\title{
Some Monodromy Representations of Generalized Braid Groups
}

\author{
A. Leibman \\ Department of Mathematics, Israel Institute of Technology - Technion, Haifa, 32000, Israel
}

Received: 1 August 1993

\begin{abstract}
A flat connection on the trivial bundle over the complement in $\mathbf{C}^{n}$ of the complexification of the system of the reflecting hyperplanes of the $B_{n}, D_{n}$ Coxeter groups is built from a simple Lie algebra and its representation. The corresponding monodromy representations of the generalized braid groups $X B_{n}, X D_{n}$ are computed in the simplest case.
\end{abstract}

\section{Introduction}

0.1. Let $\left\{l_{1}, \ldots, l_{m}\right\}$ be a hyperplane arrangement in $\mathbf{C}^{n}$, the hyperplane $l_{i}$ be defined by the equation $L_{i}\left(x_{1}, \ldots, x_{n}\right)=0, X=\mathbf{C}^{n} \backslash \bigcup_{i=1}^{m} l_{i}$. Let $\Omega_{i}, i=1, \ldots, d$ be $m \times m$ complex matrices; then the 1 -forms matrix

$$
\Omega=\sum_{i=1}^{m} \Omega_{i} \mathrm{~d} \log L_{i}
$$

defines a connection on the trivial bundle $X \times V \rightarrow X$ where the fiber $V$ is a $n$ dimensional complex vector space. The condition that the connection be flat, that is $\Omega \wedge \Omega+\mathrm{d} \Omega=0$, reads here as follows:

if $h \subset \mathbf{C}^{n}$ is a subspace of codimension 2 and $J_{h}=\left\{i \leqq d: h \subset l_{i}\right\}$, then

$$
\left[\Omega_{j}, \sum_{i \in J_{h}} \Omega_{i}\right]=0 \text { for every } j \in J_{h}
$$

(see, for example, [5]).

0.2. The flat connection on the bundle gives a monodromy representation of the fundamental group of the base of the bundle by the action on its fiber. This monodromy was thoroughly investigated in the case in which the hyperplane arrangement is the complexification of the system of the reflecting hyperplanes in $\mathbf{R}^{n} \subset \mathbf{C}^{n}$ of the Weyl group of the root system $A_{n-1}$, and the fundamental group is the pure braid group (see $[6,7]$ ). Here $L_{i, j}: x_{i}=x_{j}$ and the conditions (1) of flatness 
of the connection $\Omega=\sum_{1 \leqq i<j \leqq n} \Omega_{i, j} \mathrm{~d} \log L_{i, j}$ have the form

$$
\begin{aligned}
& {\left[\Omega_{i, j}, \Omega_{i, k}+\Omega_{j, k}\right]=0,} \\
& {\left[\Omega_{i, j}, \Omega_{k, l}\right]=0 \text { for distinct } i, j, k, l}
\end{aligned}
$$

(we put $\Omega_{i, j}=\Omega_{j, i}$ ) and are called "the infinitesimal pure braid relations."

0.3. A series of solutions of these equations ([1]) is obtained in the following way: let $g$ be a semisimple finite-dimensional complex Lie algebra, $U$ its universal enveloping algebra, $\left\{I_{p}\right\}_{1}^{m}$ an orthobasis of $g$ with respect to the Killing form, $\tau=\sum I_{p} \otimes I_{p} \in U \otimes U$. Put $\tau_{1,2}=\tau \otimes 1, \tau_{2,3}=1 \otimes \tau, \tau_{1,3}=\cdot \otimes 1 \otimes \cdot \in U \otimes U \otimes U$.

Then

$$
\left[\tau_{1,2}, \tau_{2,3}+\tau_{1,3}\right]=\left[\tau_{2,3}, \tau_{1,2}+\tau_{1,3}\right]=0 .
$$

Let $\rho: g \rightarrow \operatorname{End}(W)$ be a finite-dimensional representation of $g$; put $V=W^{\otimes n}$, $\tau_{i, j}^{\rho}=\rho_{i, j}(\tau)$, where $\rho_{i, j}=\mathbf{1}^{\otimes(i-1)} \otimes \rho \otimes \mathbf{1}^{\otimes(j-i-1)} \otimes \rho \otimes \mathbf{1}^{\otimes(n-j)}: g \otimes g \rightarrow \operatorname{End}(V)$. Because of (3), $\tau_{i, j}^{\rho}$ satisfy the infinitesimal pure braid relations (2) and define therefore a flat connection on the trivial bundle with the fiber $V$. Kohno $([6,7])$ has found the corresponding monodromy action of the pure braid group (and even of the braid group) for nonexceptional simple Lie algebras $g$.

0.4. In this paper we describe an analogous construction for the generalized pure braid group of type $B_{n}$ and, under certain restrictions, $D_{n}$, and describe the corresponding monodromy in the simplest case: $g=\mathbf{s l}_{2}(\mathbf{C})$ and $\rho$ is its standard 2-dimensional representation. The corresponding infinitesimal relations include those of $A_{n-1}$ and we start from the same construction.

Analogous problems were solved in $[2,3]$ in significantly more abstract form; we have not established whether our constructions are partial cases of those considered in these papers.

\section{Computations in Lie Algebras}

1.1. As in $[1,6,7]$, let $g$ be a semisimple finite-dimensional complex Lie algebra, $U$ its universal enveloping algebra, (,) be the Killing form, $A \subset H^{*}$ be the system of the roots of $g$ with respect to a Cartan subalgebra $H$ of $g$; denote by $\left\{e_{\alpha}\right\}_{\alpha \in A}$ a Weyl system of root vectors (see, for example, [8]): $e_{\alpha} \in \alpha,\left(e_{\alpha}, e_{-\alpha}\right)=1,\left[e_{\alpha}, e_{-\alpha}\right]=h_{\alpha} \in H$, $\left[e_{\alpha}, e_{\beta}\right]=N_{\alpha, \beta} e_{\alpha+\beta}$ for $\alpha+\beta \neq 0$ with $N_{\alpha, \beta}=N_{-\alpha,-\beta} \in \mathbf{C}$. Let $\left\{h_{i}=h_{\alpha_{i}}\right\}_{i=1}^{d}$ be a basis of $H$ over $\mathbf{C}, H_{i, j}=\left(h_{i}, h_{j}\right), G=H^{-1}$.

Denote

$$
\begin{aligned}
& \tau=\sum_{\alpha \neq 0} e_{\alpha} \otimes e_{-\alpha}+\sum_{i, j=1}^{d} G_{i, j} h_{i} \otimes h_{j} \in U \otimes U, \\
& \mu=\sum_{\alpha \neq 0} e_{\alpha} \otimes e_{\alpha}-\sum_{i, j=1}^{d} G_{i, j} h_{i} \otimes h_{j} \in U \otimes U, \\
& \nu=\frac{1}{2}\left(\sum_{\alpha \neq 0} e_{\alpha}^{2}+\sum_{\alpha \neq 0} e_{\alpha} e_{-\alpha}\right) \in U .
\end{aligned}
$$


Let $\sigma \in \operatorname{Aut}(U): \sigma\left(e_{\alpha}\right)=e_{-\alpha}, \sigma\left(h_{\alpha}\right)=-h_{\alpha} ; \chi_{i}: U \rightarrow U^{\otimes n}: \chi_{i}(u)=\mathbf{1}^{\otimes(i-1)} \otimes u \otimes \mathbf{1}^{\otimes(n-i)}$, $\chi_{i, j}: U \otimes U \rightarrow U^{\otimes n}: \chi_{i, j}(u \otimes v)=\mathbf{1}^{\otimes(i-1)} \otimes u \otimes \mathbf{1}^{\otimes(j-i-1)} \otimes v \otimes \mathbf{1}^{\otimes(n-j)} ;$

$$
\tau_{i, j}=\chi_{i, j}(\tau), \quad \mu_{i, j}=\chi_{i, j}(\mu), \quad v_{i}=\chi_{i}(v) \in U^{\otimes n} .
$$

1.2. Proposition. In the above notation,

1) $\left[\tau_{i, j}, \tau_{i, k}+\tau_{j, k}\right]=0$,

2) a) $\left[\tau_{i, j}, \mu_{i, k}+\mu_{j, k}\right]=0$, b) $\left[\mu_{i, k}, \tau_{i, j}+\mu_{j, k}\right]=0$,

3) a) $\left[\tau_{i, j}+v_{i}+v_{j}, \mu_{i, j}\right]=0$ b) $\left[\mu_{i, j}+v_{i}+v_{j}, \tau_{i, j}\right]=0$,

c) $\left[\tau_{i, j}+v_{i}+\mu_{i, j}, v_{j}\right]=0$,

(4) $\left[\tau_{i, j}, \mu_{k, l}\right]=\left[\tau_{i, j}, v_{k}\right]=\left[\mu_{i, j}, v_{k}\right]=0$,

for distinct $i, j, k, l$.

Proof.

1) Our $\tau$ coincides with Belavin-Drinfeld's: if $\left\{I_{p}\right\}_{1}^{m}$ is an orthobasis of $g$, then $\tau=\sum_{p=1}^{m} I_{p} \otimes I_{p}$, and 1) holds. Let us reproduce the proof ([6]): let $\delta: U \rightarrow U \otimes U$ : $\delta a=a \otimes 1+1 \otimes a$ for $a \in g, \quad c=\sum_{p=1}^{m} I_{p}^{2} \in U$ be the Casimir element. Then $\tau=\frac{1}{2}(\delta(\mathbf{c})-\mathbf{c} \otimes \mathbf{1}-\mathbf{1} \otimes \mathbf{c}) ;$ therefore $[\tau, \delta(u)]=0$ for every $u \in U$ and

$$
\left[\tau_{i, j}, \tau_{i, k}+\tau_{j, k}\right]=\left[\tau_{i, j}, \sum_{p=1}^{m} \chi_{i, j}\left(\delta\left(I_{p}\right)\right) \chi_{k}\left(I_{p}\right)\right]=0 .
$$

2) Note that $\mu=\left(\mathrm{id}_{U} \otimes \sigma\right)(\tau)=\left(\sigma \otimes \mathrm{id}_{U}\right)(\tau), \sigma^{2}=\mathrm{id}_{U}$. Hence,

$$
\begin{aligned}
& {\left[\tau_{i, j}, \mu_{i, k}+\mu_{j, k}\right]=\mathrm{id}_{U}^{\otimes(k-1)} \otimes \sigma \otimes \mathrm{id}_{U}^{\otimes(n-k)}\left(\left[\tau_{i, j}, \tau_{i, k}+\tau_{j, k}\right]\right)=0,} \\
& {\left[\mu_{i, k}, \tau_{i, j}+\mu_{j, k}\right]=\mathrm{id}_{U}^{\otimes(k-1)} \otimes \sigma \otimes \mathrm{id}_{U}^{\otimes(n-k)}\left(\left[\tau_{i, k}, \tau_{i, j}+\tau_{j, k}\right]\right)=0,}
\end{aligned}
$$

3) It is enough to prove that

$$
\begin{aligned}
& \text { a) }[\tau+v \otimes \mathbf{1}+\mathbf{1} \otimes v, \mu]=0, \text { b) }[\mu+v \otimes \mathbf{1}+\mathbf{1} \otimes v, \tau]=0, \\
& \left.\left.\mathrm{c}^{\prime}\right)[\tau+\mu, v \otimes \mathbf{1}]=0, \mathrm{c}^{\prime \prime}\right)[v \otimes \mathbf{1}, \mathbf{1} \otimes v]=0 .
\end{aligned}
$$

$\mathrm{c}^{\prime \prime}$ ) is evident; as $\sigma(v)=v, \mathrm{~b}$ ) follows from a). We need some computations to prove a) and $\mathrm{c}^{\prime}$ ). Remember, that $\left[e_{\alpha}, e_{\beta}\right]=N_{\alpha, \beta} e_{\alpha+\beta}$ for $\alpha, \beta \in A, \alpha+\beta \neq 0$; extend the range of this formula onto the complete lattice generated by $A$ in $H^{*}$ setting $N_{\alpha, \beta}=0$ if one of $\alpha, \beta$ or $\alpha+\beta$ does not belong to $A$. We will use the following equalities (see, for example, [8]):

$$
\begin{aligned}
{\left[e_{\alpha}, e_{-\alpha}\right] } & =h_{\alpha},\left[e_{\alpha}, h_{\alpha}\right]=-2 e_{\alpha}, h_{\alpha}=-h_{-\alpha}, \\
{\left[e_{\alpha}^{2}, e_{\beta}\right] } & =N_{\alpha, \beta} e_{\alpha} e_{\alpha+\beta}+N_{\alpha, \beta} e_{\alpha+\beta} e_{\alpha} \text { for } \beta \neq-\alpha, \\
{\left[e_{\alpha}^{2}, e_{-\alpha}\right] } & =e_{\alpha} h_{\alpha}+h_{\alpha} e_{\alpha}, \\
{\left[e_{\alpha}, h_{k}\right] } & =-\left(h_{\alpha}, h_{k}\right) e_{\alpha},\left[e_{\alpha}^{2}, h_{k}\right]=-2\left(h_{\alpha}, h_{k}\right) e_{\alpha}^{2},\left[e_{\alpha} e_{-\alpha}, h_{k}\right]=0, \\
N_{\alpha, \beta} & =-N_{\beta, \alpha}=-N_{-\alpha-\beta, \beta}=-N_{\alpha,-\alpha-\beta}=N_{-\alpha,-\beta}, N_{\alpha, \alpha}=0, \\
h_{\alpha} & =\sum_{i, j=1}^{d}\left(h_{\alpha}, h_{j}\right) G_{i, j} h_{i} .
\end{aligned}
$$


a) $\left[\sum_{\alpha \neq 0} e_{\alpha} \otimes e_{-\alpha}+\sum_{i, j=1}^{d} G_{i, j} h_{i} \otimes h_{j}+\frac{1}{2} \sum_{\alpha \neq 0} e_{\alpha}^{2} \otimes 1+\frac{1}{2} \sum_{\alpha \neq 0} e_{\alpha} e_{-\alpha} \otimes 1\right.$

$$
\begin{aligned}
& \left.+\frac{1}{2} \sum_{\alpha \neq 0} 1 \otimes e_{\alpha}^{2}+\frac{1}{2} \sum_{\alpha \neq 0} 1 \otimes e_{\alpha} e_{-\alpha}, \sum_{\beta \neq 0} e_{\beta} \otimes e_{\beta}-\sum_{k, l=1}^{d} G_{k, l} h_{k} \otimes h_{l}\right] \\
& =\sum_{\substack{\alpha \neq 0 \\
\beta \neq 0}}\left[e_{\alpha}, e_{\beta}\right] \otimes e_{-\alpha} e_{\beta}+\sum_{\substack{\alpha \neq 0 \\
\beta \neq 0}} e_{\beta} e_{\alpha} \otimes\left[e_{-\alpha}, e_{\beta}\right] \\
& +\sum_{\substack{i, j=1 \\
\beta \neq 0}}^{d} G_{i, j}\left[h_{i}, e_{\beta}\right] \otimes h_{j} e_{\beta}+\sum_{\substack{i, j=1 \\
\beta \neq 0}}^{d} G_{i, j} e_{\beta} h_{i} \otimes\left[h_{j}, e_{\beta}\right] \\
& +\frac{1}{2} \sum_{\substack{\alpha \neq 0 \\
\beta \neq 0}}\left[e_{\alpha}^{2}, e_{\beta}\right] \otimes e_{\beta}+\frac{1}{2} \sum_{\substack{\alpha \neq 0 \\
\beta \neq 0}}\left[e_{\alpha} e_{-\alpha}, e_{\beta}\right] \otimes e_{\beta}+\frac{1}{2} \sum_{\substack{\alpha \neq 0 \\
\beta \neq 0}} e_{\beta} \otimes\left[e_{\alpha}^{2}, e_{\beta}\right] \\
& +\frac{1}{2} \sum_{\substack{\alpha \neq 0 \\
\beta \neq 0}} e_{\beta} \otimes\left[e_{\alpha} e_{-\alpha}, e_{\beta}\right]-\sum_{\substack{k, l=1 \\
\alpha \neq 0}}^{d} G_{k, l}\left[e_{\alpha}, h_{k}\right] \otimes e_{-\alpha} h_{l} \\
& -\sum_{\substack{k, l=1 \\
\alpha \neq 0}}^{d} G_{k, l} h_{k} e_{\alpha} \otimes\left[e_{-\alpha}, h_{l}\right]-\frac{1}{2} \sum_{\substack{k, l=1 \\
\alpha \neq 0}}^{d} G_{k, l}\left[e_{\alpha}^{2}, h_{k}\right] \otimes h_{l}
\end{aligned}
$$$$
-\frac{1}{2} \sum_{\substack{k, l=1 \\ \alpha \neq 0}}^{d} G_{k, l}\left[e_{\alpha} e_{-\alpha}, h_{k}\right] \otimes h_{l}-\frac{1}{2} \sum_{\substack{k, l=1 \\ \alpha \neq 0}}^{d} G_{k, l} h_{k} \otimes\left[e_{\alpha}^{2}, h_{l}\right]
$$$$
-\frac{1}{2} \sum_{\substack{k, l=1 \\ \alpha \neq 0}}^{d} G_{k, l} h_{k} \otimes\left[e_{\alpha} e_{-\alpha}, h_{k}\right]=\sum_{\substack{\alpha \neq 0 \\ \beta \neq 0 \\ \alpha+\beta \neq 0}} N_{\alpha, \beta} e_{\alpha+\beta} \otimes e_{-\alpha} e_{\beta}
$$$$
+\sum_{\alpha \neq 0} h_{\alpha} \otimes e_{-\alpha}^{2}+\sum_{\substack{\alpha \neq 0 \\ \beta \neq 0 \\ \alpha-\beta \neq 0}} N_{-\alpha, \beta} e_{\beta} e_{\alpha} \otimes e_{-\alpha+\beta}+\sum_{\alpha \neq 0} e_{\alpha}^{2} \otimes h_{-\alpha}
$$$$
+\sum_{\substack{i, j=1 \\ \beta \neq 0}}^{d} G_{i, j}\left(h_{i}, h_{\beta}\right) e_{\beta} \otimes h_{j} e_{\beta}+\sum_{\substack{i, j=1 \\ \beta \neq 0}}^{d} G_{i, j}\left(h_{j}, h_{\beta}\right) e_{\beta} h_{i} \otimes e_{\beta}
$$$$
+\frac{1}{2} \sum_{\substack{\alpha \neq 0 \\ \beta \neq 0 \\ \alpha+\beta \neq 0}} N_{\alpha, \beta} e_{\alpha+\beta} e_{\alpha} \otimes e_{\beta}+\frac{1}{2} \sum_{\alpha \neq 0} h_{\alpha} e_{\alpha} \otimes e_{-\alpha}+\frac{1}{2} \sum_{\substack{\alpha \neq 0 \\ \beta \neq 0 \\ \alpha \neq \beta \neq 0}} N_{\alpha, \beta} e_{\alpha} e_{\alpha+\beta} \otimes e_{\beta}
$$

$$
\begin{gathered}
\left.+\frac{1}{2} \sum_{\alpha \neq 0} e_{\alpha} h_{\alpha} \otimes e_{-\alpha}+\frac{1}{2} \sum_{\substack{\alpha \neq 0 \\
\beta \neq 0 \\
\left(S_{10}\right)}} N_{\alpha+\beta \neq 0} e_{\alpha+\beta} e_{-\alpha} \otimes e_{\beta}+\frac{1}{2} \sum_{\alpha \neq 0} h_{\alpha} e_{-\alpha} \otimes e_{-\alpha}\right) \\
+\frac{1}{2} \sum_{\substack{\alpha \neq 0 \\
\beta \neq 0 \\
\alpha-\beta \neq 0}} N_{-\alpha, \beta} e_{\alpha} e_{-\alpha+\beta} \otimes e_{\beta}+\frac{1}{2} \sum_{\alpha \neq 0} e_{\alpha} h_{-\alpha} \otimes e_{\alpha} \\
\left(S_{13}\right) \\
\left(S_{14}\right)
\end{gathered}
$$




$$
\begin{aligned}
& +\frac{1}{2} \sum_{\substack{\alpha \neq 0 \\
\beta \neq 0 \\
\alpha+\beta \neq 0}} N_{\alpha, \beta} e_{\beta} \otimes e_{\alpha+\beta} e_{\alpha}+\frac{1}{2} \sum_{\alpha \neq 0} e_{-\alpha} \otimes h_{\alpha} e_{\alpha}+\frac{1}{2} \sum_{\substack{\alpha \neq 0 \\
\beta \neq 0 \\
\alpha+\beta \neq 0}} N_{\alpha, \beta} e_{\beta} \otimes e_{\alpha} e_{\alpha+\beta} \\
& +\frac{1}{2} \sum_{\alpha \neq 0} e_{-\alpha} \otimes e_{\alpha} h_{\alpha}+\frac{1}{2} \sum_{\substack{\alpha \neq 0 \\
\beta \neq 0 \\
\alpha+\beta \neq 0}} N_{\substack{\alpha, \beta \\
\left(S_{18}\right)}} N_{\beta} \otimes e e_{\alpha+\beta} e_{-\alpha}+\frac{1}{2} \sum_{\alpha \neq 0} e_{-\alpha} \otimes h_{\alpha} e_{-\alpha} \\
& +\frac{1}{2} \sum_{\substack{\alpha \neq 0 \\
\beta \neq 0 \\
\alpha-\beta \neq 0}} N_{-\alpha, \beta} e_{\beta} \otimes e_{\alpha} e_{-\alpha+\beta}+\frac{1}{2} \sum_{\alpha \neq 0} e_{\alpha} \otimes e_{\alpha} h_{-\alpha}\left(S_{22}\right) \\
& +\sum_{\substack{k, l=1 \\
\alpha \neq 0}}^{d} G_{k, l}\left(h_{\alpha}, h_{k}\right) e_{\alpha} \otimes e_{-\alpha} h_{l}+\sum_{\substack{k, l=1 \\
\alpha \neq 0}}^{d} G_{k, l}\left(h_{-\alpha}, h_{l}\right) h_{k} e_{\alpha} \otimes e_{-\alpha} \\
& +\sum_{\substack{k, l=1 \\
\alpha \neq 0}}^{d} G_{k, l}\left(h_{\alpha}, h_{k}\right) e_{\alpha}^{2} \otimes h_{l}+\sum_{\substack{k, l=1 \\
\alpha \neq 0}}^{d} G_{k, l}\left(h_{25}, h_{l}\right) h_{k} \otimes e_{\alpha}^{2} \\
& S_{5}+\frac{1}{2} S_{20}+\frac{1}{2} S_{22}=\sum_{\substack{i, j=1 \\
\beta \neq 0}}^{d} G_{i, j}\left(h_{i}, h_{\beta}\right) e_{\beta} \otimes h_{j} e_{\beta}+\frac{1}{2} \sum_{\alpha \neq 0} e_{-\alpha} \otimes h_{\alpha} e_{-\alpha} \\
& +\frac{1}{2} \sum_{\alpha \neq 0} e_{\alpha} \otimes e_{\alpha} h_{-\alpha}=\sum_{\beta \neq 0} e_{\beta} \otimes h_{\beta} e_{\beta}-\sum_{\alpha \neq 0} e_{\alpha} \otimes h_{\alpha} e_{\alpha} \\
& +\frac{1}{2} \sum_{\alpha \neq 0} e_{\alpha} \otimes\left[e_{\alpha}, h_{-\alpha}\right]=\sum_{\alpha \neq 0} e_{\alpha} \otimes e_{\alpha}, \\
& S_{6}+\frac{1}{2} S_{12}+\frac{1}{2} S_{14}=\sum_{\substack{i, j=1 \\
\beta \neq 0}}^{d} G_{i, j}\left(h_{j}, h_{\beta}\right) e_{\beta} h_{i} \otimes e_{\beta}+\frac{1}{2} \sum_{\alpha \neq 0} h_{\alpha} e_{-\alpha} \otimes e_{-\alpha} \\
& +\frac{1}{2} \sum_{\alpha \neq 0} e_{\alpha} h_{-\alpha} \otimes e_{\alpha}=\sum_{\beta \neq 0} e_{\beta} h_{\beta} \otimes e_{\beta}-\sum_{\alpha \neq 0} e_{\alpha} h_{\alpha} \otimes e_{\alpha} \\
& +\frac{1}{2} \sum_{\alpha \neq 0}\left[h_{\alpha}, e_{-\alpha}\right] \otimes e_{-\alpha}=-\sum_{\alpha \neq 0} e_{\alpha} \otimes e_{\alpha},
\end{aligned}
$$

So, $S_{5}+\frac{1}{2} S_{20}+\frac{1}{2} S_{22}+S_{6}+\frac{1}{2} S_{12}+\frac{1}{2} S_{14}=0$.

$$
\begin{aligned}
S_{23}+\frac{1}{2} S_{16}+\frac{1}{2} S_{18}= & \sum_{\substack{k, l=1 \\
\alpha \neq 0}}^{d} G_{k, l}\left(h_{\alpha}, h_{k}\right) e_{\alpha} \otimes e_{-\alpha} h_{l}+\frac{1}{2} \sum_{\alpha \neq 0} e_{-\alpha} \otimes h_{\alpha} e_{\alpha} \\
& +\frac{1}{2} \sum_{\alpha \neq 0} e_{-\alpha} \otimes e_{\alpha} h_{\alpha}=\sum_{\alpha \neq 0} e_{\alpha} \otimes e_{-\alpha} h_{\alpha}-\sum_{\alpha \neq 0} e_{\alpha} \otimes e_{-\alpha} h_{\alpha} \\
& +\frac{1}{2} \sum_{\alpha \neq 0} e_{-\alpha} \otimes\left[h_{\alpha}, e_{\alpha}\right]=\sum_{\alpha \neq 0} e_{-\alpha} \otimes e_{\alpha},
\end{aligned}
$$




$$
\begin{aligned}
S_{24}+\frac{1}{2} S_{8}+\frac{1}{2} S_{10}= & \sum_{\substack{k, l=1 \\
\alpha \neq 0}}^{d} G_{k, l}\left(h_{-\alpha}, h_{l}\right) h_{k} e_{\alpha} \otimes e_{-\alpha}+\frac{1}{2} \sum_{\alpha \neq 0} h_{\alpha} e_{\alpha} \otimes e_{-\alpha} \\
& +\frac{1}{2} \sum_{\alpha \neq 0} e_{\alpha} h_{\alpha} \otimes e_{-\alpha}=-\sum_{\alpha \neq 0} h_{\alpha} e_{\alpha} \otimes e_{\alpha}+\sum_{\alpha \neq 0} h_{\alpha} e_{\alpha} \otimes e_{-\alpha} \\
& +\frac{1}{2} \sum_{\alpha \neq 0}\left[e_{\alpha}, h_{\alpha}\right] \otimes e_{-\alpha}=-\sum_{\alpha \neq 0} e_{-\alpha} \otimes e_{-\alpha},
\end{aligned}
$$

So, $S_{23}+\frac{1}{2} S_{16}+\frac{1}{2} S_{18}+S_{24}+\frac{1}{2} S_{8}+\frac{1}{2} S_{10}=0$.

$S_{25}+S_{4}=\sum_{\substack{k, l=1 \\ \alpha \neq 0}}^{d} G_{k, l}\left(h_{\alpha}, h_{k}\right) e_{\alpha}^{2} \otimes h_{l}+\sum_{\alpha \neq 0} e_{\alpha}^{2} \otimes h_{-\alpha}=\sum_{\alpha \neq 0} e_{\alpha}^{2} \otimes h_{\alpha}-\sum_{\alpha \neq 0} e_{\alpha}^{2} \otimes h_{\alpha}=0$

$S_{26}+S_{2}=\sum_{\substack{k, l=1 \\ \alpha \neq 0}}^{d} G_{k, l}\left(h_{\alpha}, h_{l}\right) h_{k} \otimes e_{\alpha}^{2}+\sum_{\alpha \neq 0} h_{\alpha} \otimes e_{-\alpha}^{2}=\sum_{\alpha \neq 0} h_{\alpha} \otimes e_{\alpha}^{2}-\sum_{\alpha \neq 0} h_{-\alpha} \otimes e_{-\alpha}^{2}=0$.

Denote $\alpha^{\prime}=\alpha-\beta$.

$$
\begin{aligned}
S_{13} & =\sum_{\substack{\alpha \neq 0 \\
\beta \neq 0 \\
\alpha-\beta \neq 0}} N_{-\alpha, \beta} e_{\alpha} e_{-\alpha+\beta} \otimes e_{\beta}=\sum_{\substack{\alpha^{\prime} \neq 0 \\
\beta \neq 0 \\
\alpha^{\prime}+\beta \neq 0}} N_{-\alpha^{\prime}-\beta, \beta} e_{\alpha^{\prime}+\beta} e_{-\alpha^{\prime}} \otimes e_{\beta} \\
= & -\sum_{\substack{\alpha^{\prime} \neq 0 \\
\beta \neq 0 \\
\alpha^{\prime}+\beta \neq 0}} N_{\alpha^{\prime}, \beta} e_{\alpha^{\prime}+\beta} e_{-\alpha^{\prime}} \otimes e_{\beta}=-S_{11} .
\end{aligned}
$$

So, $S_{11}+S_{13}=0$.

$$
\begin{aligned}
S_{21} & =\sum_{\substack{\alpha \neq 0 \\
\beta \neq 0 \\
\alpha-\beta \neq 0}} N_{-\alpha, \beta} e_{\beta} \otimes e_{\alpha} e_{-\alpha+\beta}=\sum_{\begin{array}{c}
\alpha^{\prime} \neq 0 \\
\beta \neq 0 \\
\alpha^{\prime}+\beta \neq 0
\end{array}} N_{-\alpha^{\prime}-\beta, \beta} e_{\beta} \otimes e_{\alpha^{\prime}+\beta} e_{-\alpha^{\prime}} \\
= & -\sum_{\substack{\alpha^{\prime} \neq 0 \\
\beta \neq 0 \\
\alpha^{\prime}+\beta \neq 0}} N_{\alpha^{\prime}, \beta} e_{\beta} \otimes e_{\alpha^{\prime}+\beta} e_{-\alpha^{\prime}}=-S_{19} .
\end{aligned}
$$

So, $S_{19}+S_{21}=0$.

Denote $\beta^{\prime}=\alpha+\beta, \alpha^{\prime}=-\alpha$.

$$
\begin{aligned}
S_{15} & =\sum_{\substack{\alpha \neq 0 \\
\beta \neq 0 \\
\alpha+\beta \neq 0}} N_{\alpha, \beta} e_{\beta} \otimes e_{\alpha+\beta} e_{\alpha}=\sum_{\substack{\alpha \neq 0 \\
\beta^{\prime} \neq 0 \\
\alpha-\beta^{\prime} \neq 0}} N_{\alpha,-\alpha+\beta^{\prime}} e_{-\alpha+\beta} \otimes e_{\beta}, e_{\alpha} \\
& =\sum_{\substack{\alpha \neq 0 \\
\beta^{\prime} \neq 0 \\
\alpha-\beta^{\prime} \neq 0}} N_{\alpha,-\alpha+\beta^{\prime}} e_{-\alpha+\beta^{\prime}} \otimes e_{\alpha} e_{\beta^{\prime}}+\sum_{\substack{\alpha \neq 0 \\
\beta^{\prime} \neq 0 \\
\alpha-\beta^{\prime} \neq 0}} N_{\alpha,-\alpha+\beta^{\prime}} e_{-\alpha+\beta^{\prime}} \otimes\left[e_{\beta^{\prime}}, e_{\alpha}\right]
\end{aligned}
$$




$$
\begin{aligned}
& =-\sum_{\substack{\alpha \neq 0 \\
\beta^{\prime} \neq 0 \\
\alpha-\beta^{\prime} \neq 0}} N_{\alpha,-\beta^{\prime}} e_{-\alpha+\beta^{\prime}} \otimes e_{\alpha} e_{\beta^{\prime}}-\sum_{\substack{\alpha \neq 0 \\
\beta^{\prime} \neq 0 \\
\alpha+\beta^{\prime} \neq 0 \\
\alpha-\beta^{\prime} \neq 0}} N_{\alpha,-\beta^{\prime}} N_{\beta^{\prime}, \alpha} e_{-\alpha+\beta^{\prime}} \otimes e_{\alpha+\beta^{\prime}} \\
& \quad-\sum_{\substack{\alpha \neq 0 \\
\beta^{\prime}}} N_{\alpha, \alpha} e_{-2 \alpha} \otimes h_{-\alpha}=-\sum_{\substack{\alpha \neq 0 \\
\beta^{\prime} \neq 0 \\
\alpha+\beta^{\prime} \neq 0}} N_{\alpha^{\prime}, \beta^{\prime}} e_{\alpha^{\prime}+\beta^{\prime}} \otimes e_{-\alpha^{\prime}} e_{\beta^{\prime}} \\
& +\sum_{\substack{\alpha \neq \beta^{\prime} \neq 0 \\
\alpha-\beta^{\prime} \neq 0}} N_{\alpha,-\beta^{\prime}} N_{\alpha, \beta^{\prime}} e_{-\alpha+\beta^{\prime}} \otimes e_{\alpha+\beta^{\prime}}, \\
& S_{17}=\sum_{\substack{\alpha \neq 0 \\
\beta \neq 0 \\
\alpha+\beta \neq 0}} N_{\alpha, \beta} e_{\beta} \otimes e_{\alpha} e_{\alpha+\beta}=\sum_{\substack{\alpha \neq 0 \\
\beta^{\prime} \neq 0 \\
\alpha-\beta^{\prime} \neq 0}} N_{\alpha,-\alpha+\beta^{\prime}} e_{-\alpha+\beta^{\prime}} \otimes e_{\alpha} e_{\beta^{\prime}} \\
& =-\sum_{\substack{\alpha \neq 0 \\
\beta^{\prime} \neq 0 \\
\alpha+\beta^{\prime} \neq 0}} N_{\alpha^{\prime}, \beta^{\prime}} e_{\alpha^{\prime}+\beta^{\prime}} \otimes e_{-\alpha^{\prime}} e_{\beta^{\prime}} .
\end{aligned}
$$

So,

$$
\begin{aligned}
& S_{1}+\frac{1}{2} S_{15}+\frac{1}{2} S_{17} \\
& =\sum_{\substack{\alpha \neq 0 \\
\beta \neq 0 \\
\alpha+\beta \neq 0}} N_{\alpha, \beta} e_{\alpha+\beta} \otimes e_{-\alpha} e_{\beta}-\frac{1}{2} \sum_{\substack{\alpha \neq 0 \\
\beta \neq 0 \\
\alpha+\beta \neq 0}} N_{\alpha, \beta} e_{\alpha+\beta} \otimes e_{-\alpha} e_{\beta} \\
& +\frac{1}{2} \sum_{\substack{\alpha \neq 0 \\
\beta \neq 0 \\
\alpha+\beta \neq 0 \\
\alpha-\beta \neq 0}} N_{\alpha,-\beta} N_{\alpha, \beta} e_{-\alpha+\beta} \otimes e_{\alpha+\beta}-\frac{1}{2} \sum_{\substack{\alpha \neq 0 \\
\beta \neq 0 \\
\alpha+\beta \neq 0}} N_{\alpha, \beta} e_{\alpha+\beta} \otimes e_{-\alpha} e_{\beta} \\
& =\frac{1}{2} \sum_{\begin{array}{c}
\alpha^{\prime} \neq 0 \\
\beta \neq 0 \\
\alpha^{\prime}+\beta \neq 0 \\
\alpha^{\prime}-\beta \neq 0
\end{array}} N_{-\alpha^{\prime},-\beta} N_{-\alpha^{\prime}, \beta} e_{\alpha^{\prime}+\beta} \otimes e_{-\alpha^{\prime}+\beta}=\frac{1}{2} \sum_{\begin{array}{c}
\alpha^{\prime} \neq 0 \\
\beta \neq 0 \\
\alpha^{\prime}+\beta \neq 0 \\
\alpha^{\prime}-\beta \neq 0
\end{array}} N_{\alpha^{\prime}, \beta} N_{\alpha^{\prime},-\beta} e_{\alpha^{\prime}+\beta} \otimes e_{-\alpha^{\prime}+\beta} . \\
& S_{9}=\sum_{\substack{\alpha \neq 0 \\
\beta \neq 0 \\
\alpha+\beta \neq 0}} N_{\alpha, \beta} e_{\alpha} e_{\alpha+\beta} \otimes e_{\beta}=\sum_{\substack{\alpha \neq 0 \\
\beta^{\prime} \neq 0 \\
\alpha^{\prime}-\beta^{\prime} \neq 0}} N_{\alpha,-\alpha+\beta^{\prime}} e_{\alpha} e_{\beta^{\prime}} \otimes e_{-\alpha+\beta^{\prime}} \\
& =\sum_{\substack{\alpha^{\prime} \neq 0 \\
\beta^{\prime} \neq 0 \\
\alpha^{\prime}-\beta^{\prime} \neq 0}} N_{\alpha,-\alpha+\beta^{\prime}} e_{\beta^{\prime}} e_{\alpha} \otimes e_{-\alpha+\beta^{\prime}}+\sum_{\begin{array}{r}
\alpha^{\prime} \neq 0 \\
\beta^{\prime} \neq 0 \\
\alpha^{\prime}-\beta^{\prime} \neq 0
\end{array}} N_{\alpha,-\alpha+\beta^{\prime}}\left[e_{\alpha}, e_{\beta^{\prime}}\right] \otimes e_{-\alpha+\beta^{\prime}} \\
& =-\sum_{\substack{\alpha \neq 0 \\
\beta^{\prime} \neq 0 \\
\alpha-\beta^{\prime} \neq 0}} N_{\alpha,-\beta^{\prime}} e_{\beta^{\prime}} e_{\alpha} \otimes e_{-\alpha+\beta^{\prime}}-\sum_{\substack{\alpha \neq 0 \\
\beta^{\prime} \neq 0 \\
\alpha+\beta^{\prime} \neq 0 \\
\alpha-\beta^{\prime} \neq 0}} N_{\alpha,-\beta^{\prime}} N_{\alpha, \beta^{\prime}} e_{\alpha+\beta^{\prime}} \otimes e_{-\alpha+\beta^{\prime}} \\
& -\sum_{\alpha \neq 0} N_{\alpha, \alpha} h_{\alpha} \otimes e_{-2 \alpha},
\end{aligned}
$$




$$
\begin{aligned}
& S_{7}= \sum_{\substack{\alpha \neq 0 \\
\beta \neq 0 \\
\alpha+\beta \neq 0}} N_{\alpha, \beta} e_{\alpha+\beta} e_{\alpha} \otimes e_{\beta}=\sum_{\substack{\alpha \neq 0 \\
\beta \neq 0 \\
\alpha-\beta^{\prime} \neq 0}} N_{\alpha,-\alpha+\beta^{\prime}} e_{\beta^{\prime}} e_{\alpha} \otimes e_{-\alpha+\beta^{\prime}} \\
&=-\sum_{\substack{\alpha \neq 0 \\
\beta^{\prime} \neq 0 \\
\alpha-\beta^{\prime} \neq 0}} N_{-\alpha, \beta^{\prime}} e_{\beta^{\prime}} e_{\alpha} \otimes e_{-\alpha+\beta^{\prime}} .
\end{aligned}
$$

So,

$$
\begin{aligned}
& S_{3}+\frac{1}{2} S_{7}+\frac{1}{2} S_{9} \\
& =\sum_{\substack{\alpha \neq 0 \\
\beta \neq 0 \\
\alpha-\beta \neq 0}} N_{-\alpha, \beta} e_{\beta} e_{\alpha} \otimes e_{-\alpha+\beta}-\frac{1}{2} \sum_{\substack{\alpha \neq 0 \\
\beta \neq 0 \\
\alpha-\beta \neq 0}} N_{-\alpha, \beta} e_{\beta} e_{\alpha} \otimes e_{-\alpha+\beta} \\
& \quad-\frac{1}{2} \sum_{\substack{\alpha \neq 0 \\
\beta \neq 0}} N_{-\alpha, \beta} e_{\beta} e_{\alpha} \otimes e_{-\alpha+\beta}-\frac{1}{2} \sum_{\substack{\alpha \neq 0 \\
\beta \neq 0 \\
\alpha+\beta \neq 0 \\
\alpha-\beta \neq 0}} N_{\alpha,-\beta} N_{\alpha, \beta} e_{\alpha+\beta} \otimes e_{-\alpha+\beta} \\
& =-\frac{1}{2} \sum_{\substack{\alpha \neq 0 \\
\beta \neq 0 \\
\alpha+\beta \neq 0 \\
\alpha-\beta \neq 0}} N_{\alpha,-\beta} N_{\alpha, \beta} e_{\alpha+\beta} \otimes e_{-\alpha+\beta} \cdot .
\end{aligned}
$$

Hence, $S_{1}+\frac{1}{2} S_{15}+\frac{1}{2} S_{17}+S_{3}+\frac{1}{2} S_{7}+\frac{1}{2} S_{9}=0$.

$\left.\mathrm{c}^{\prime}\right) \quad 2[\mu+\tau, v \otimes \mathbf{1}]=\left[\sum_{\alpha \neq 0} e_{\alpha} \otimes e_{\alpha}+\sum_{i, j=1}^{d} G_{i, j} h_{i} \otimes h_{j}+\sum_{\alpha \neq 0} e_{-\alpha} \otimes e_{\alpha}\right.$

$$
\begin{gathered}
\left.-\sum_{i, j=1}^{d} G_{i, j} h_{i} \otimes h_{j},\left(\sum_{\beta \neq 0}\left(e_{\beta}^{2}+e_{\beta} e_{-\beta}\right)\right) \otimes 1\right] \\
=\sum_{\alpha \neq 0}\left[\left(e_{\alpha}+e_{-\alpha}\right), \sum_{\beta \neq 0}\left(e_{\beta}^{2}+e_{\beta} e_{-\beta}\right)\right] \otimes e_{\alpha} . \\
{\left[e_{\alpha}+e_{-\alpha}, \sum_{\beta \neq 0}\left(e_{\beta}^{2}+e_{\beta} e_{-\beta}\right)\right]=\sum_{\beta \neq 0}\left(\left[e_{\alpha}, e_{\beta}\right] e_{\beta}+e_{\beta}\left[e_{\alpha}, e_{\beta}\right]+\left[e_{\alpha}, e_{\beta}\right] e_{-\beta}\right.} \\
\left.+e_{\beta}\left[e_{\alpha}, e_{-\beta}\right]+\left[e_{-\alpha}, e_{\beta}\right] e_{\beta}+e_{\beta}\left[e_{-\alpha}, e_{\beta}\right]+\left[e_{-\alpha}, e_{\beta}\right] e_{-\beta}+e_{\beta}\left[e_{-\alpha}, e_{-\beta}\right]\right) \\
\left.=\sum_{\substack{\beta \neq 0 \\
\beta \neq-\alpha}} N_{\alpha, \beta} e_{\alpha+\beta} e_{\beta}+h_{\alpha} e_{-\alpha}+\sum_{\substack{\beta \neq 0 \\
\beta \neq-\alpha}} N_{\alpha, \beta} e_{\beta} e_{\alpha+\beta}+e_{-\alpha} h_{\alpha}\right) \\
+\sum_{\substack{\beta \neq 0 \\
\beta \neq-\alpha}} N_{\alpha, \beta} e_{\alpha+\beta} e_{-\beta}+h_{\alpha} e_{\alpha}+\sum_{\substack{\beta \neq 0 \\
\beta \neq \alpha}} N_{\alpha,-\beta}\left(S_{\beta} e_{\alpha-\beta}+e_{\alpha} h_{\alpha}\right. \\
+\sum_{\substack{\beta \neq 0 \\
\beta \neq \alpha}} N_{-\alpha, \beta} e_{-\alpha+\beta} e_{\beta}+h_{-\alpha} e_{\alpha}+\sum_{\substack{\beta \neq 0 \\
\beta \neq \alpha}} N_{-\alpha, \beta}\left(S_{6} e_{-\alpha+\beta}+e_{\alpha} h_{-\alpha}\right. \\
+\sum_{\substack{\beta \neq 0 \\
\beta \neq \alpha}} N_{-\alpha, \beta} e_{-\alpha+\beta} e_{-\beta}+h_{-\alpha} e_{-\alpha}+\sum_{\substack{\beta \neq 0 \\
\beta \neq-\alpha}} N_{-\alpha,-\beta} e_{\beta} e_{-\alpha-\beta}+e_{-\alpha} h_{-\alpha} . \\
\left(S_{7}\right)
\end{gathered}
$$


As $h_{\alpha}=-h_{-\alpha}$, the terms of the forms $e_{ \pm \alpha} h_{ \pm \alpha}, h_{ \pm \alpha} e_{ \pm \alpha}$ cancel out in the last expression. Furthermore, putting $\beta^{\prime}=\beta+\alpha$, we have

$$
\begin{aligned}
S_{1} & =\sum_{\substack{\beta \neq 0 \\
\beta \neq-\alpha}} N_{\alpha, \beta} e_{\alpha+\beta} e_{\beta}=\sum_{\substack{\beta^{\prime} \neq 0 \\
\beta^{\prime} \neq \alpha}} N_{\alpha,-\alpha+\beta^{\prime}} e_{\beta^{\prime}} e_{-\alpha+\beta^{\prime}}=-\sum_{\substack{\beta^{\prime} \neq 0 \\
\beta^{\prime} \neq \alpha}} N_{\alpha,-\beta^{\prime}} e_{\beta^{\prime}} e_{-\alpha+\beta^{\prime}} \\
& =-\sum_{\substack{\beta^{\prime} \neq 0 \\
\beta^{\prime} \neq \alpha}} N_{-\alpha, \beta^{\prime}} e_{\beta^{\prime}} e_{-\alpha+\beta^{\prime}}=-S_{6} .
\end{aligned}
$$

Similarly, $S_{2}=-S_{5}, S_{3}=-S_{4}, S_{8}=-S_{7}$, and our expression is equal to zero. 4) is evident.

\section{The Monodromy}

2.1. Let $W$ be a finite-dimensional space $\rho: g \rightarrow \operatorname{End}(W)$ be a representation of $g$, and $V=W^{\otimes n}$. The hyperplane arrangement of the root system $B_{n}$ is given by the equations $x_{i} \pm x_{j}=0, x_{i}=0$; define a connection on the trivial bundle $\mathbf{C}^{n} \times V$ over its complement $X \subset \mathbf{C}^{n}$ by the 1 -form matrix,

$$
\begin{aligned}
\Omega= & \lambda\left(\sum_{1 \leqq i<j \leqq n} \rho_{n}\left(\tau_{i, j}\right) \mathrm{d} \log \left(x_{i}-x_{j}\right)+\sum_{1 \leqq i<j \leqq n} \rho_{n}\left(\mu_{i, j}\right) \mathrm{d} \log \left(x_{i}+x_{j}\right)\right. \\
& \left.+\sum_{1 \leqq i \leqq n} \rho_{n}\left(v_{i}\right) \mathrm{d} \log \left(x_{i}\right)\right),
\end{aligned}
$$

where $\lambda \in \mathbf{C}, \tau_{i, j}, \mu_{i, j}, v_{i, j}$ are defined by (4), (5), and $\rho_{n}: U^{\otimes n} \rightarrow \operatorname{End}\left(V=W^{\otimes n}\right)$ is the representation of $U^{\otimes n}$ constructed from $\rho: \rho_{n}\left(g_{1} \otimes \cdots \otimes g_{n}\right)=$ $\rho\left(g_{1}\right) \otimes \cdots \otimes \rho\left(g_{n}\right)$.

Proposition (1.2) says that this connection is flat and, consequently, it defines a representation of the generalized pure braid group $P B_{n}=\pi_{1}(X)$ in $\operatorname{End}(V)$.

2.2. In the case when $g=\mathbf{s l}_{m}(\mathbf{C})$ and $\rho$ is its standard representation, we have also a flat connection over the complement $X^{\prime}$ of the hyperplane arrangement $D_{n}: x_{i} \pm x_{j}=0$ in $\mathbf{C}^{n}$, due to the following fact:

Lemma. In the above assumptions, $\rho(v)$ is proportional to $\mathrm{id}_{W}$, and, consequently, $\left[\tau_{i, j}, \mu_{i, j}\right]=0$.

Proof. If we put $H$ to be the diagonal matrices in the standard representation of $\mathbf{s l}_{m}(\mathbf{C})$, then the matrix corresponding to $e_{i, j}$ has a nonzero element only in the $(i, j)$ place; $\rho\left(e_{i, j}^{2}\right)=0, \rho\left(e_{i, j} e_{j, i}\right)$ is diagonal, so $\rho(v)$ is diagonal as well and is invariant under permutations of the elements of the basis of $W$.

Hence, the matrix

$$
\Omega^{\prime}=\lambda\left(\sum_{1 \leqq i<j \leqq n} \rho_{n}\left(\tau_{i, j}\right) \mathrm{d} \log \left(x_{i}-x_{j}\right)+\sum_{1 \leqq i<j \leqq n} \rho_{n}\left(\mu_{i, j}\right) \mathrm{d} \log \left(x_{i}+x_{j}\right)\right)
$$

defines a flat connection on $X^{\prime}$ and a monodromy representation of the generalized pure braid group $P D_{n}=\pi_{1}\left(X^{\prime}\right)$. 
2.3. We are going to describe these monodromies in the simplest case: from now on, $g=s l_{2}(\mathbf{C})$, and $\rho$ is its standard representation

$$
\rho\left(e_{+}\right)=\left(\begin{array}{ll}
0 & 1 \\
0 & 0
\end{array}\right), \quad \rho\left(e_{-}\right)=\left(\begin{array}{ll}
0 & 0 \\
1 & 0
\end{array}\right), \quad \rho(h)=\left(\begin{array}{rr}
1 & 0 \\
0 & -1
\end{array}\right)
$$

in a basis $\left\{e_{1}, e_{2}\right\}$ of a 2 -dimensional vector space $W$.

Change for this case the definition (4) of $v$ by setting

$$
v=r\left(\sum_{\alpha \neq 0} e_{\alpha}^{2}+\sum_{\alpha \neq 0} e_{\alpha} e_{-\alpha}\right)+s\left(\sum_{\alpha \neq 0} e_{\alpha}\right) .
$$

Lemma. The equalities (6) hold for the new $v$ as well.

Proof. The equalities

$$
\left[\tau, \sum_{\alpha \neq 0} e_{\alpha} \otimes 1+\sum_{\alpha \neq 0} 1 \otimes e_{\alpha}\right]=0, \quad\left[\mu, \sum_{\alpha \neq 0} e_{\alpha} \otimes 1+\sum_{\alpha \neq 0} 1 \otimes e_{\alpha}\right]=0
$$

hold for all semisimple $g$, it is seen from the proof of Proposition 1.2 a); we have to check only, that $\left[e_{+} \otimes e_{-}+e_{-} \otimes e_{+}+e_{+} \otimes e_{+}+e_{-} \otimes e_{-},\left(e_{+}+e_{-}\right) \otimes 1\right]$ is equal to 0 . But it is $\left[e_{+}+e_{-}, e_{+}+e_{-}\right] \otimes\left(e_{+}+e_{-}\right)=0$.

2.4. Let $a_{1}, \ldots, a_{n-1}, c_{1}$ be the generators of the generalized braid group $X B_{n}$ of the root system $B_{n}$ and, simultaneously, of the Weyl group $W B_{n}$ corresponding to the reflections with respect to the hyperplanes $\left\{x_{1}=x_{2}\right\}, \ldots,\left\{x_{n-1}=x_{n}\right\},\left\{x_{1}=0\right\}$ respectively. $X B_{n}$ is described by the relations

$$
\begin{array}{ll}
a_{i} a_{j}=a_{j} a_{i} & \text { for }|i-j| \geqq 2, a_{i} a_{i+1} a_{i}=a_{i+1} a_{i} a_{i+1}, \\
c_{1} a_{i}=a_{i} c_{1} & \text { for } i \geqq 2,\left(c_{1} a_{1}\right)^{2}=\left(a_{1} c_{1}\right)^{2} ;
\end{array}
$$

$W B_{n}$ has in addition the relations $a_{1}^{2}=\ldots=a_{n-1}^{2}=c_{1}^{2}=1$. The groups $X D_{n}$ and $W D_{n}$, corresponding to the system $D_{n}$, are generated by the elements $a_{1}, \ldots, a_{n-1}$, $b_{1}$ with the relations

$$
\begin{array}{ll}
a_{i} a_{j}=a_{j} a_{i} & \text { for }|i-j| \geqq 2, a_{i} a_{i+1} a_{i}=a_{i+1} a_{i} a_{i+1}, \\
b_{1} a_{i}=a_{i} b_{1} & \text { for } i \neq 2, b_{1} a_{2} b_{1}=a_{2} b_{1} a_{2},
\end{array}
$$

and $W D_{n}$ has in addition the relations, $a_{1}^{2}=\ldots a_{n-1}^{2}=b_{1}^{2}=\mathbf{1}$.

2.5. Denote $q=e^{-\pi \sqrt{-1} \lambda / 4}$.

Theorem. The monodromy $\theta_{p}: P B_{n} \rightarrow \operatorname{End}(V)$ defined by the connection (7) is, up to conjugation, the restriction of the representation $\tilde{\theta}$ of the group $X B_{n}$ given by the matrices

$$
\begin{aligned}
& \tilde{\theta}\left(a_{i}\right)=q^{-1 / 2} \mathbf{1}^{\otimes(i-1)} \otimes\left(\begin{array}{cccc}
1 & 0 & 0 & 0 \\
0 & 0 & q & 0 \\
0 & q & 1-q^{2} & 0 \\
0 & 0 & 0 & 1
\end{array}\right) \otimes \mathbf{1}^{\otimes(n-i-1)}, \\
& \tilde{\theta}\left(c_{1}\right)=q^{-r}\left(\begin{array}{cc}
0 & 1 \\
1 & q^{-2 s}-q^{2 s}
\end{array}\right) \otimes \mathbf{1}^{\otimes(n-1)},
\end{aligned}
$$


where the matrices singled out are given in the basis $\left\{e_{1} \otimes e_{1}, e_{2} \otimes e_{1}, e_{1} \otimes e_{2}\right.$, $\left.e_{2} \otimes e_{2}\right\}$ of $W \otimes W$ and $\left\{e_{1}, e_{2}\right\}$ of $W$ respectively. The monodromy $\theta_{P}^{\prime}: P D_{n} \rightarrow \operatorname{End}(V)$ defined by the connection (8) is, up to conjugation, the restriction of the representation $\tilde{\theta}^{\prime}$ of the group $X B_{n}$ given by the matrices

$$
\tilde{\theta}^{\prime}\left(a_{i}\right)=\tilde{\theta}\left(a_{i}\right), \tilde{\theta}^{\prime}\left(b_{1}\right)=q^{-1 / 2}\left(\begin{array}{cccc}
0 & 0 & 0 & q \\
0 & 1 & 0 & 0 \\
0 & 0 & 1 & 0 \\
q & 0 & 0 & 1-q^{2}
\end{array}\right) \otimes \mathbf{1}^{\otimes(n-2)} .
$$

Proof is modification of that of Theorem 3.2.3 of [6].

Define an action of the Weyl group $W B_{n}$ on $V=W^{\otimes n}$ simultaneously with its action on $X$ so that the connection be invariant under the product action on $X \times V$. Namely, put

$$
\begin{gathered}
a_{i}\left(v_{1} \otimes \cdots \otimes v_{i} \otimes v_{i+1} \otimes \cdots \otimes v_{n}\right)=v_{1} \otimes \cdots \otimes v_{i+1} \otimes v_{i} \otimes \cdots \otimes v_{n}, \\
c_{1}\left(e_{k} \otimes v_{2} \otimes \cdots \otimes v_{n}\right)=e_{3-k} \otimes v_{2} \otimes \cdots \otimes v_{n}, k=1,2 .
\end{gathered}
$$

Indeed, in terms of the action of $U^{\otimes n}$ on $V, c_{1}^{-1} \rho_{n} c_{1}=\rho_{n} \circ\left(\sigma \otimes 1^{\otimes(n-1)}\right)$ and, therefore, $c_{1}^{-1} \rho_{n}\left(\tau_{1,2}\right) c_{1}=\rho_{n}\left(\mu_{1,2}\right)$. After we quotient $X \times V$ by this action, we will obtain a (non-trivial) bundle over $X / W B_{n}$ having $V$ as a fiber and endowed with a flat connection. The fundamental group of $X / W B_{n}$ is the generalized braid group $X B_{n}$, hence the monodromy of the flat connection yields a representation $\theta$ of $X / W B_{n}$ in $\operatorname{End}(V)$, whose restriction $\left.\theta\right|_{P B_{n}}$ on pure braids is $\theta_{P}$. It depends of the parameter $\lambda$; when $\lambda=0$ the action of $X B_{n}$ on $V$ coincides with the action (13) of $W B_{n}$.

The monodromy action on $V$ of the simple loop half bypassing the hyperplane $\left\{x_{i}=x_{j}\right\}$ in $X$, that is $\theta\left(a_{i}\right)$, is defined by the residue of $\Omega$ along this hyperplane $\lambda \rho_{n}\left(\tau_{i, i+1}\right)$ and coincides, up to conjugation, with the composition of the exponent of this matrix $e^{\pi \sqrt{-1} \lambda \rho_{n}\left(\tau_{l, i+1}\right)}$ and the action (13) of $a_{i}$. The matrix has the form

$$
\mathbf{1}^{\otimes(i-1)} \otimes\left(\begin{array}{cccc}
1 / 8 & 0 & 0 & 0 \\
0 & -1 / 8 & 1 / 4 & 0 \\
0 & 1 / 4 & -1 / 8 & 0 \\
0 & 0 & 0 & 1 / 8
\end{array}\right) \otimes \mathbf{1}^{\otimes(n-i-1)} ;
$$

it is semisimple with the set of eigenvalues $\{1 / 8,-3 / 8\}$, the eigenvectors corresponding to the second one lying in the subspace $W^{\otimes(i-1)} \otimes\left(e_{1} \otimes e_{2}-\right.$ $\left.e_{2} \otimes e_{1}\right) \otimes W^{\otimes(n-i-1)}$ of $V$. Its exponent is semisimple with the set of eigenvalues $\left\{e^{\pi \sqrt{-1} \lambda 1 / 8}, e^{\pi \sqrt{-1} \lambda(-3 / 8)}\right\}$, and $a_{i}$ from (13) acts as -1 on the subspace corresponding to the second eigenvalue and as $\mathbf{1}$ on the subspace corresponding to the first one. Hence, $\theta\left(a_{i}\right)$ is semisimple with the eigenvalues $\left\{q^{-1 / 2}=e^{\pi \sqrt{-1} \lambda(1 / 8)}\right.$, $\left.-q^{3 / 2}=-e^{\pi \sqrt{-1} \lambda(-3 / 8)}\right\}$ and satisfies the quadratic equation

$$
\left(\theta\left(a_{i}\right)-q^{-1 / 2}\right)\left(\theta\left(a_{i}\right)+q^{3 / 2}\right)=0 .
$$



matrix

In the same way, the residue of $\Omega$ along the hyperplane $\left\{x_{1}=0\right\}$ is given by the

$$
\left(\begin{array}{ll}
r / 4 & s / 2 \\
s / 2 & r / 4
\end{array}\right) \otimes 1^{\otimes(n-1)}
$$

with the eigenvalues $r / 4+s / 2, r / 4-s / 2$ and $\theta\left(c_{1}\right)$ satisfy the equation

$$
\left(\theta\left(c_{1}\right)-q^{-r-2 s}\right)\left(\theta\left(c_{1}\right)+q^{-r+2 s}\right)=0 .
$$

Let $A B_{n}$ be the maximal algebra over $\mathbf{C}$ generated by the elements $a_{1}, \ldots, a_{n-1}, c_{1}$ in which the relations (9) and, in addition, the following relations coming from (14), (15) hold:

$$
a_{i}^{2}=q^{-1 / 2}\left(1-q^{2}\right) a_{i}+q, \quad c_{1}^{2}=q^{-r-2 s}\left(1-q^{4 s}\right) c_{1}+q^{-2 r} .
$$

This algebra is obtained by a deformation with parameter $q$ from the group algebra $\mathbf{C} W B_{n}$, which corresponds to $q=1$ (that is, to $\lambda=0$ ), and has the same dimension: there exists a natural way to reduce the elements of $\mathbf{C} W B_{n}$ to a natural form without augmentation of their lengths computed in the alphabet $\left\{a_{1}, \ldots, a_{n-1}, c_{1}\right\}$; we can use the same process to reduce the elements of $A B_{n}$ to the same form if we replace the relations $a_{i}^{2}=c_{1}^{2}=1$ of $\mathbf{C} W B_{n}$ by (16). Hence, the algebra $A B_{n}$ is semisimple for small $\lambda$ and the decomposition of any of its representation into irreducible ones depends continuously on $\lambda$ if $\lambda$ is small enough (see [4]).

The representations $\theta, \tilde{\theta}$ satisfy Eqs. (14), (15) and factorize through $A B_{n}$ (for $\tilde{\theta}$ this is verified directly). When $\lambda=0$, both of them coincide with (13). Hence, these representations are equivalent for small $\lambda$. But they depend on $\lambda$ analytically and, therefore, $\theta$ is equivalent to $\tilde{\theta}$ for any $\lambda$.

The second part of the theorem, dealing with the representation of $X D_{n}$, is proved in the same way.

Acknowledgement. I am grateful to Dr. D. Markushevich for his advice and help during my work on this paper.

\section{References}

1. Belavin, A.A., Drinfeld, V.G.: Solutions of the classical Yang-Baxter equations for simple Lie algebras. Funct. Anal. Appl. 16, 1-29 (1982)

2. Cherednik, I.V.: Calculation of the monodromy of some $W$-invariant local systems of type $B$, $C$ and D. Funct. Anal. Appl. 24, 1, 78-79 (1990)

3. Cherednik, I.V.: Generalized braid groups and local $r$-matrix systems. Soviet Math. Dokl. 40,1, 43-48 (1990)

4. Jones, V.: Hecke algebra representations of braid groups and link polynomials. Ann. of Math. 126, 335-338 (1987)

5. Kohno, T.: On the holonomy Lie algebra and the nilpotent completion of the fundamental group of the complement of hypersurfaces. Nagoya Math. J. 92, 21-37 (1983)

6. Kohno, T.: Linear representations of braid groups and classical Yang-Baxter equations. Contemp. Math. 78, 339-362 (1988)

7. Kohno, T.: Monodromy representations of braid groups and Yang-Baxter equations. Ann. Inst. Fourier, Grenoble 37, 4, 139-160 (1987)

8. Varadarajan, V.S.: Lie Groups, Lie Algebras, and Their Representations. Berlin, Heidelberg, New York: Springer 1984 